\title{
Comparison of preference and safety of powder and liquid lactulose in adult patients with chronic constipation
}

This article was published in the following Dove Press journal:

Clinical and Experimental Gastroenterology

19 October 2010

Number of times this article has been viewed

\author{
Charles F Barish' \\ Bryan Voss ${ }^{2}$ \\ Byron Kaelin² \\ 'Wake Research Associates, Raleigh, \\ North Carolina, USA; ${ }^{2}$ Cumberland \\ Pharmaceuticals Inc., Nashville, \\ Tennessee, USA
}

Background: Chronic constipation is an important clinical condition which can result in serious discomfort and even require hospitalization. Powder and liquid lactulose are designated as clinically equivalent for the treatment of constipation, but there are significant differences in the taste, consistency, and portability of the products, which may affect patient compliance and therefore clinical outcome.

Aim: To evaluate patient preference between powder and liquid lactulose in terms of overall preference, taste, consistency, and portability, and safety in terms of adverse events.

Methods: Three sites randomized patients (total $n=50$ ) to powder or liquid lactulose for seven days with crossover. Patient preference was assessed by a questionnaire, and the occurrence of adverse events was monitored.

Results: Of those expressing a preference, $44 \%$ and $57 \%$ more patients preferred the taste and consistency, respectively, of powder over liquid lactulose. More than six times as many patients preferred the portability of powder compared with liquid lactulose and, overall, 77\% more patients preferred powder over liquid lactulose. There was no difference between treatment groups in terms of adverse events $(P=0.635)$.

Conclusions: More patients preferred powder compared with liquid lactulose and the products were equally safe. These findings may impact patient compliance, and therefore may affect clinical outcome.

Keywords: constipation, lactulose, laxative

\section{Introduction}

Chronic constipation is an important clinical condition which can result in serious discomfort and decreased quality of life, and can even require hospitalization. ${ }^{1,2}$ Chronic constipation affects an estimated $15 \%$ of the North American population and has a wide range of underlying causes, from dehydration, to opioid use, to medical conditions such as endocrine, gastrointestinal, and neurologic disorders. ${ }^{2}$ Many pharmacologic, both over-the-counter and prescription, and herbal laxatives are available for the treatment of chronic constipation. ${ }^{2}$ However, a recent systematic review of the literature found that only lactulose and polyethylene glycol consistently and repeatedly loosened stools and thereby relieved constipation. ${ }^{3}$ Therefore, a prescription osmotic laxative like lactulose is a common therapeutic option for the treatment of chronic constipation. ${ }^{2,4}$

Lactulose is available in a dry, powder form (Kristalose ${ }^{\circledR}$, lactose for oral solution) to be dissolved in water and a liquid/syrup form. While the products are designated as clinically equivalent, there are notable differences in the taste, consistency, and portability between the products. We hypothesized that these differences could result in a
Correspondence: Charles F Barish, MD Wake Research Associates, 3100

Duraleigh Road, Raleigh, NC 276I2, USA

$\mathrm{Tel}+191978 \mid 2514$

Fax +I 9194206067

Email cfbgastro@aol.com 
difference in patient preference between powder and liquid lactulose. Increased patient preference can correlate with increased patient compliance. It is well known that decreased patient compliance results in poor patient outcomes. ${ }^{5,6}$ Additionally, a significant number of patients with hepatic encephalopathy require daily treatment with large amounts of liquid lactulose (more than $50 \mathrm{~mL}$ per day) and these patients are often noncompliant because they are unable to ingest and/or keep down this large amount of lactulose syrup due to its taste and consistency. ${ }^{7-9}$ Therefore, we designed and conducted a study to determine whether patients prefer powder or liquid lactulose in terms of overall preference, taste, consistency, and portability. The safety of the products was also assessed.

\section{Methods}

\section{Patients}

This clinical trial was a prospective, randomized, open-label, multicenter, seven-day, crossover study. Patients seen at the outpatient clinics of Wake Research Associates (Raleigh, NC, USA), Rapid Medical Research (Cleveland, OH, USA), and Arya Gastroenterology (Brooklyn, NY, USA) with a recent diagnosis of chronic constipation (within the last 90 days) were eligible for enrollment in this study. Patients provided written informed consent before enrollment, and the study protocol was approved by the Western Institutional Review Board and the study was registered on ClinicalTrials. gov (NCT00712543). Inclusion criteria included a recent diagnosis of chronic constipation (within the last 90 days). Exclusion criteria included patients with galactosemia (galactose-sensitive diet), patients younger than 18 years of age, patients currently on lactulose therapy, and patients unable to understand the requirements of the study or unwilling to provide written informed consent and agreement to abide by the study restrictions.

\section{Study randomization, design, and medications}

Patients were randomized in a 1:1 ratio to receive powder or liquid lactulose first for seven days (dose determined by treating physician, range for powder $=10-20 \mathrm{~g} /$ day, range for liquid $=15-30 \mathrm{~mL} /$ day), and then the patients crossed over to the alternative treatment for the following seven days (dose again determined by treating physician, Figure 1). Patients returned to the study site on study day 6 or 7 to pick up the formulation of lactulose which they were scheduled to cross over to for the remainder of the study (Figure 1). Sealed envelopes containing the patient randomization scheme were provided to the study sites. Powder lactulose $\left(\right.$ Kristalose $^{\circledR}$,

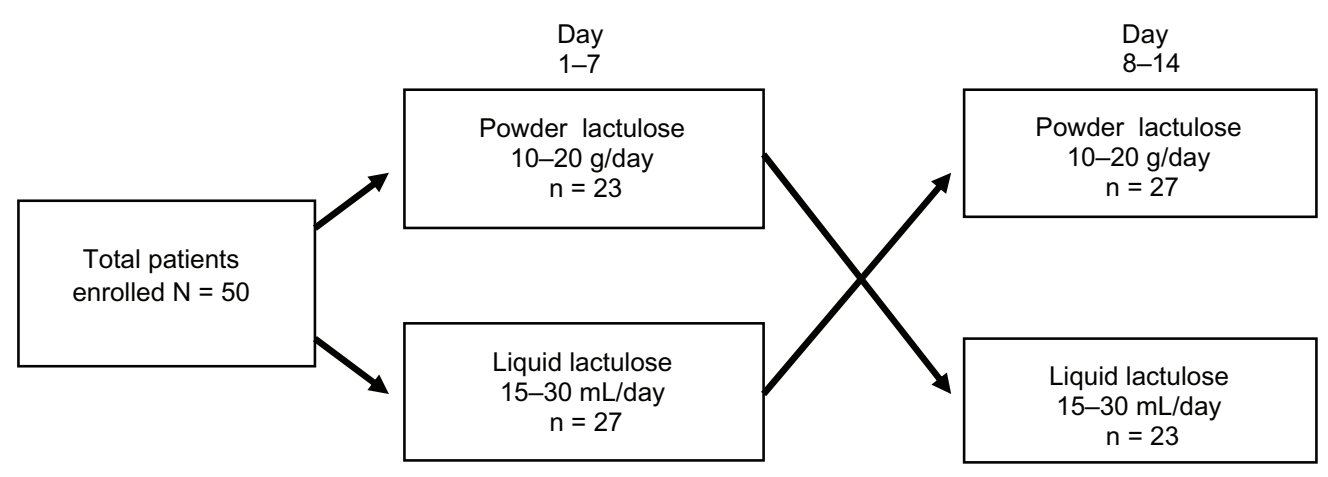

Screening/Baseline visit

(Pre-treatment period)
Study completion visit

(Post-treatment period)

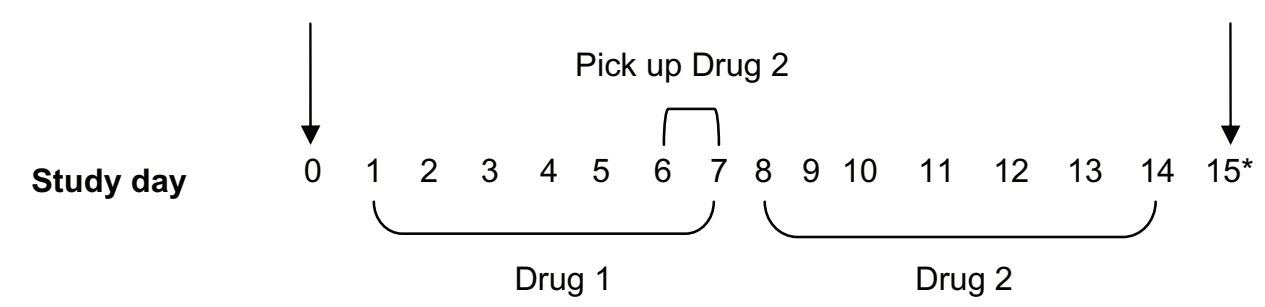

Figure I Study randomization and design. Note: *Or within 7 days of day 14 of the study. 
Cumberland Pharmaceuticals Inc., Nashville, TN, USA) was provided in $10 \mathrm{~g}$ pouches and liquid lactulose syrup (Generlac, Morton Grove Pharmaceuticals Inc., Morton Grove, IL, USA) was provided in a $473 \mathrm{~mL}$ (pint) bottle. Patient preference was assessed on a questionnaire administered by study staff in the days following completion of the study. Both treatment groups received both drugs in a crossover design (drug sequence determined by randomization), and then preference was evaluated after patients had been exposed to both treatments in this prospective study. The study sponsor developed the patient questionnaire and two versions were developed and utilized equally to avoid bias. One version of the questionnaire listed powder first as an answer selection for all of the questions, while the other listed liquid first as an answer selection for all of the questions. Each question on the questionnaire had three possible answers, ie, liquid, powder, or no preference. Spanish translations of the questionnaire and other study documents were made available at the request of the Brooklyn, NY site. Upon completion of the study, patients were allowed a sevenday window (total study duration including questionnaire visit $=21$ days) to return to the study site and complete the questionnaire, and adverse events were monitored throughout the entire study period (Figure 1).

\section{Study objectives}

The primary objective of this study was to determine overall patient preference for the powder or liquid form of lactulose. Secondary objectives included determination of patient preference for the powder or liquid form of lactulose in terms of taste, consistency, and portability, as well as safety in terms of the incidence of adverse events.

\section{Statistical analysis}

The responses on the questionnaire were summarized for each treatment sequence and then combined for an overall assessment. For each question, the Mainland-Gart test was used to test whether subjects preferred one product over the other product. The Mainland-Gart test excludes patients who show "no preference" ( $P$ value 1, Table 2$) \cdot{ }^{10,11}$ A second analysis method was performed for each question on the questionnaire using the Prescott test to determine whether subjects preferred one product over the other product. ${ }^{12}$ The Prescott test includes patients who show "no preference" ( $P$ value 2 , Table 2). ${ }^{12}$ The Mainland-Gart test and the Prescott test were performed side by side to assess the robustness of the data, more specifically in terms of how the inclusion of the subjects who had "no preference" affected the inference regarding which drug is preferred. Adverse events were summarized based on the treatment (powder or liquid lactulose) the subject was taking at the time of the onset of the event (Table 3 ). Fisher's Exact test was used to test whether there was a significant difference in the number of adverse events between the two treatment groups (Table 3).

\section{Results \\ Patient characteristics}

A total of 50 patients with a recent diagnosis of chronic constipation were enrolled in this study. Twenty-three of the 50 patients received powder lactulose for the first seven days followed by liquid lactulose on the following seven days, while 27 of the 50 patients first received liquid lactulose for seven days followed by powder lactulose on the following seven days. Two of the patients failed to return to the study site to complete the questionnaire, but limited safety data were still available for these patients. The study group demographics are shown in Table 1. The mean (standard deviation, SD) age of the patients was 49 (15) years, with a female-to-male ratio of 2.85 to 1 (Table 1 ). The majority of the patients were African-American, with Caucasians, Hispanics, and Asians making up smaller portions of the study population (Table 1). The mean height of the study population was $167(10.4) \mathrm{cm}$ and the mean weight was 87.4 (21.0) kg (Table 1).

Of the 50 patients enrolled in the study, seven protocol deviations were recorded in six $(12 \%)$ patients. Protocol

Table I Patient demographics

\begin{tabular}{ll}
\hline Age (years) & 50 \\
$\mathrm{n}$ & $48.6 \mathrm{I}(\mathrm{I} 4.95)$ \\
Mean (SD) & 48.5 \\
Median & $20.75,8 \mathrm{I} .17$ \\
Minimum, maximum & \\
Gender & $13(26 \%)$ \\
Male & $37(74 \%)$ \\
Female & \\
Race & $13(26 \%)$ \\
Caucasian & $26(52 \%)$ \\
African-American & $9(18 \%)$ \\
Hispanic & $1(2 \%)$ \\
Asian & $\mathrm{I}(2 \%)$ \\
Other & \\
Height (cm) & $167.18(\mathrm{I} 0.4 \mathrm{I})$ \\
Mean (SD) & 165.95 \\
Median & $142.2,19 \mathrm{I.4}$ \\
Minimum, maximum & \\
Weight (kg) & $87.42(20.98)$ \\
Mean (SD) & $8 \mathrm{I} .55$ \\
Median & $54.4,142.8$ \\
Minimum, maximum & \\
\hline Abbreviation: SD, stan & \\
\hline
\end{tabular}

Abbreviation: SD, standard deviation. 
Table 2 Patient preference data in terms of overall preference and preference of taste, consistency, and portability

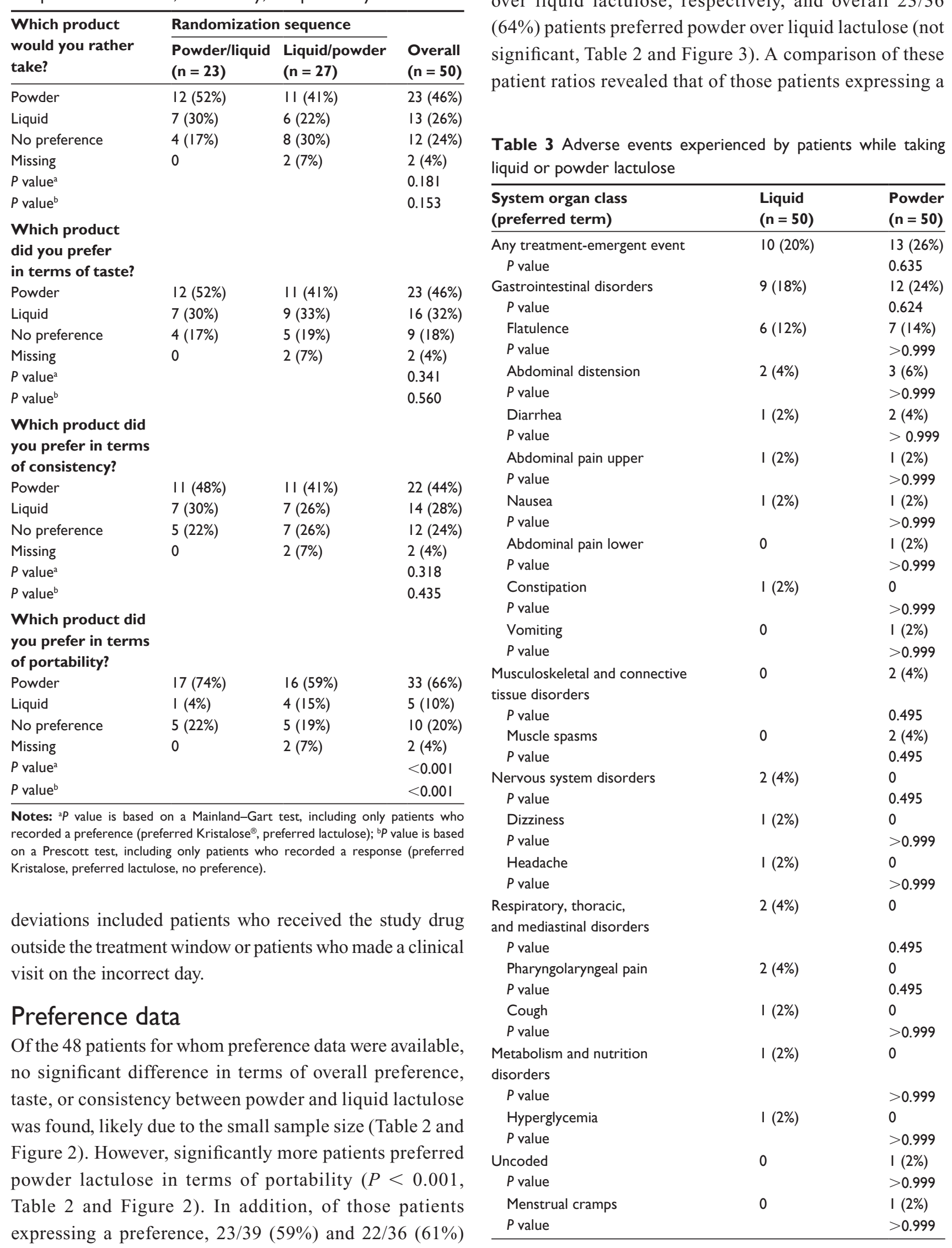

patients preferred the taste and consistency of powder over liquid lactulose, respectively, and overall 23/36 (64\%) patients preferred powder over liquid lactulose (not significant, Table 2 and Figure 3). A comparison of these patient ratios revealed that of those patients expressing a

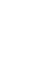




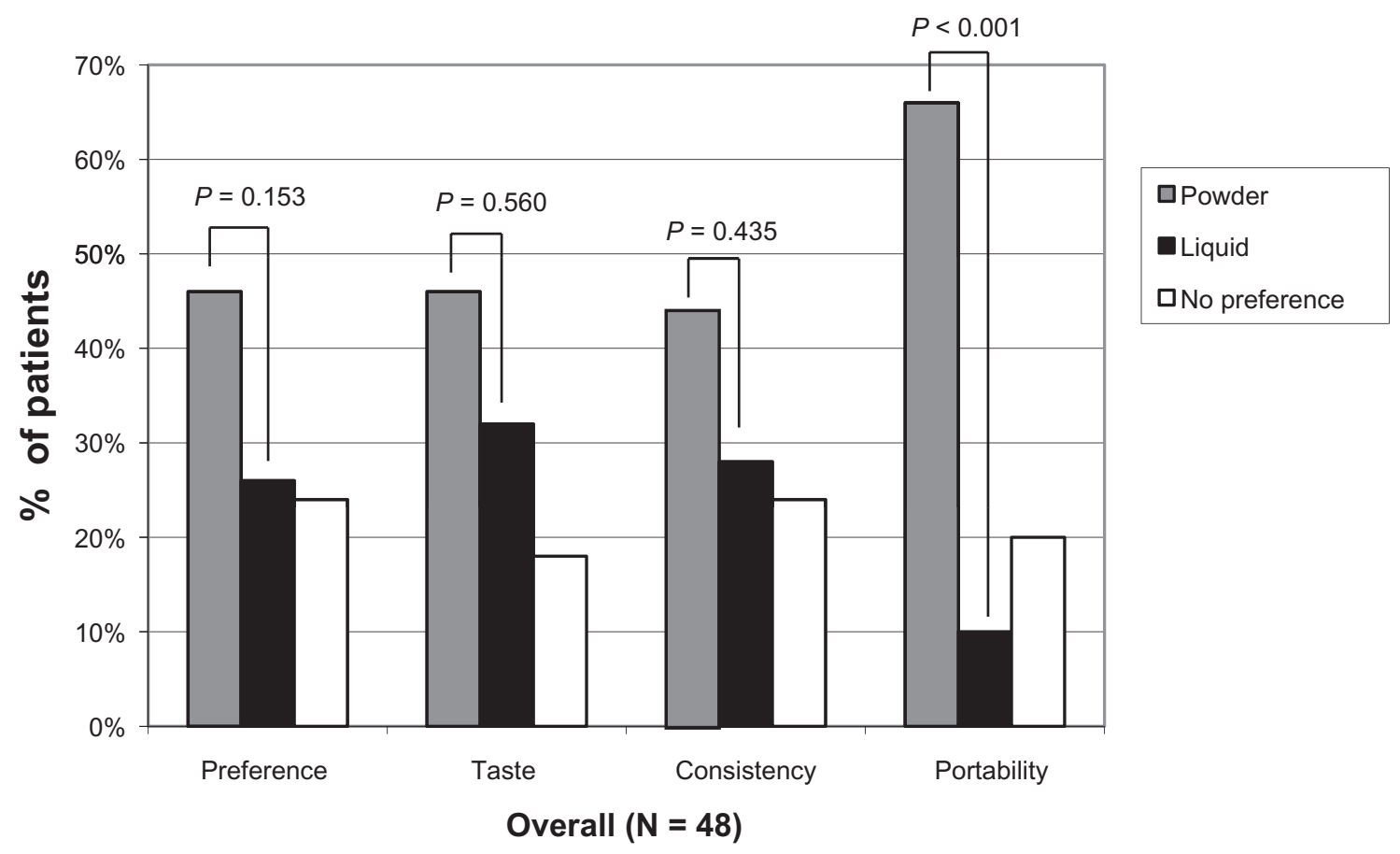

Figure 2 Preference data in terms of overall preference and preference of taste, consistency, and portability in all patients (includes those who expressed 'no preference').

preference, $44 \%$ and $57 \%$ more patients preferred the taste and consistency of powder over liquid lactulose, respectively, and overall $77 \%$ more patients preferred powder over liquid lactulose. In addition, more than six times as many patients whom expressed a preference preferred the portability of powder over liquid lactulose $(P<0.001$, Table 2 and Figure 3).

\section{Safety data}

Adverse events were of mild to moderate intensity, and ranged from gastrointestinal disorders, such as flatulence and diarrhea, to muscle spasms and pharyngolaryngeal pain. There were no statistically significant differences between treatment groups in terms of adverse event occurrence, given that 13 (26\%) patients experienced 21 adverse events

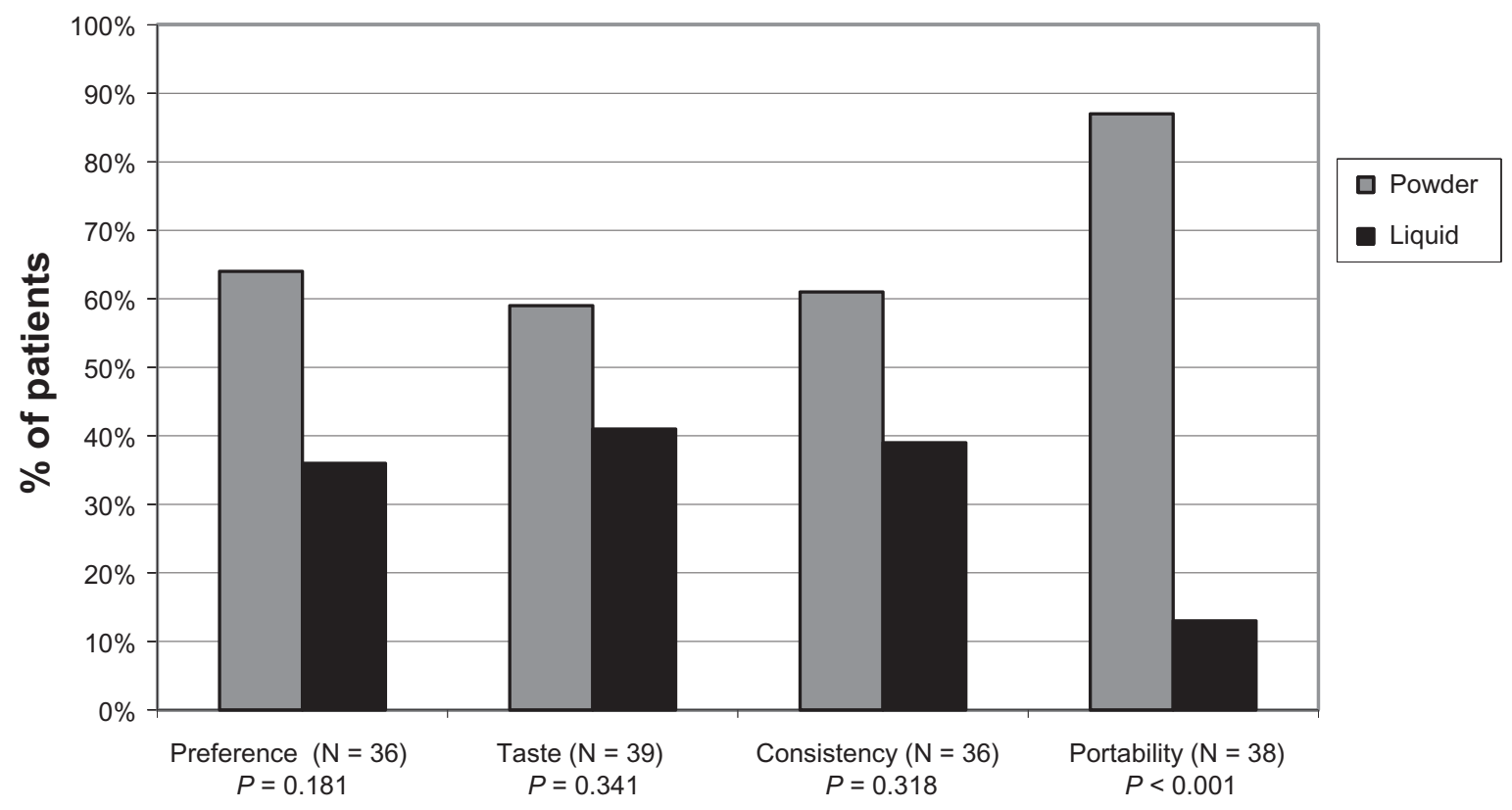

Figure 3 Preference data in terms of overall preference and preference of taste, consistency, and portability in only the patients who expressed a preference for powder or liquid lactulose. 
while taking powder lactulose and $10(20 \%)$ patients experienced 18 adverse events while taking liquid lactulose ( $P=0.635$, for the number of patients experiencing one or more adverse events, Table 3). Additionally, there were no significant differences between treatment groups for any adverse event subtype (Table 3).

\section{Discussion}

The main objective of this study was to determine whether patients prefer to take powder or liquid lactulose. Although the study was somewhat underpowered, the data showed a numeric difference in favor of powder lactulose being preferred considerably more than liquid lactulose in terms of overall preference and preference of taste and consistency. In addition, significantly more patients preferred the portability of powder lactulose compared with liquid lactulose. Powder and liquid lactulose were also found to be equally safe, because there was not a significant difference in the incidence of adverse events between treatment groups.

This study had several limitations. As was already mentioned, the study was underpowered due to the small sample size and due to three possible outcomes on the preference questionnaire (preference of powder, preference of liquid, or no preference). In addition, the study was limited geographically to the eastern US, and a broader involvement of sites may have affected the outcome of the study. The study may have also been too short in duration, as a longer dosing regimen may have altered the outcome of the study. Patients may have also had a pre-existing preference for one formulation or the other based on being previously treated with either or both of the products, but this was not factored into the study. Lastly, this study did not use standardized dosing, because the study doses were instead determined by the treating physician. For example, one patient may have been prescribed $20 \mathrm{~g}$ of powder lactulose per day compared with $15 \mathrm{~mL}$ of liquid lactulose (10 g powder lactulose $=15 \mathrm{~mL}$ liquid lactulose $)$, which may have affected patient preference.

Overall, more patients with chronic constipation preferred powder lactulose compared with liquid lactulose and the products were equally safe. Most importantly, these findings may impact patient compliance, and therefore may effect clinical outcome and thereby determine whether additional medical attention may be required.

\section{Disclosure}

This study was funded in full by Cumberland Pharmaceuticals Inc. BV and BK are employees of Cumberland Pharmaceuticals Inc. CFB has no financial interests regarding this work.

\section{References}

1. Johanson JF, Kralstein J. Chronic constipation: A survey of the patient perspective. Aliment Pharmacol Ther. 2007;25(5):599-608.

2. Gallagher P, O'Mahony D. Constipation in old age. Best Pract Res Clin Gastroenterol. 2009;23(6):875-887.

3. American College of Gastroenterology Chronic Constipation Task Force. An evidence-based approach to the management of chronic constipation in North America. Am J Gastroenterol. 2005; 100 Suppl 1: $\mathrm{S} 1-\mathrm{S} 4$.

4. Petticrew M, Rodgers M, Booth A. Effectiveness of laxatives in adults. Qual Health Care. 2001;10(4):268-273.

5. Swartz ML. Citrucel (methylcellulose/bulk-forming laxative). Gastroenterol Nurs. 1989;12(1):50-52.

6. Loening-Baucke V. Polyethylene glycol without electrolytes for children with constipation and encopresis. J Pediatr Gastroenterol Nutr. 2002;34(4):372-377.

7. Leevy CB, Phillips JA. Hospitalizations during the use of rifaximin versus lactulose for the treatment of hepatic encephalopathy. Dig Dis Sci. 2007;52(3):737-741.

8. Sundaram V, Shaikh OS. Hepatic encephalopathy: Pathophysiology and emerging therapies. Med Clin North Am. 2009;93(4):819-836, vii.

9. Generlac (lactulose for oral solution) [Product labeling]. Morton Grove, IL: Morton Grove Pharmaceuticals Inc; 2004.

10. Mainland D. Elementary Medical Statistics. Philadelphia, PA: WB Saunders; 1963.

11. Gart JJ. An exact test for comparing matched proportions in cross-over designs. Biometrika. 1969;56(1):75-80.

12. Prescott RJ. The comparison of success rates in cross-over trials in the presence of an order effect. Appl Stat. 1981;30(1):9-15.
Clinical and Experimental Gastroenterology

\section{Publish your work in this journal}

Clinical and Experimental Gastroenterology is an international, peerreviewed, open access journal, publishing all aspects of gastroenterology in the clinic and laboratory, including: Pathology, pathophysiology of gastrointestinal disease; Investigation and treatment of gastointestinal disease; Pharmacology of drugs used in the alimentary tract;

\section{Dovepress}

Immunology/genetics/genomics related to gastrointestinal disease. This journal is indexed on CAS. The manuscript management system is completely online and includes a very quick and fair peer-review system. Visit http://www.dovepress.com/testimonials.php to read real quotes from published authors. 